\title{
Thank you, Fabio Pulizzi
}

\author{
We reflect on the legacy that our long-serving Chief Editor leaves behind, as he moves on to pastures new.
}

$\Lambda$ scholarly journal, like any editorial product, is shaped by the personality, interests and character of their editors. The Chief Editor sets the priorities for the journal as they see fit, informed by their instinct, sensibility as well as data in order to serve their community.

In the past nine and a half years, our Chief Editor - Fabio Pulizzi — has predicated a close connection with the research community; he shaped a journal that is receptive to the stimulations coming from the fascinating small world of nanoscience and nanotechnology. 'Small', as in, ten to the minus nine metres; certainly not in terms of community size. The number of practitioners of the science at the nanometre scale has grown considerably since Fabio took the leading role in the journal, in June 2012. Since then, the number of articles published worldwide with the term 'nano' in the abstract has roughly doubled, as per a quick Web of Science search. But the nanoscience community has not only got bigger, it has also got more self-aware in its tools to approach real-life problems. Under Fabio's editorship, the journal has grown with the community and its scope similarly expanded.

We have observed an increased attention to applied research with substantial inroads into engineering solutions and commercial applications. This has been reflected in the rising number of submissions in the applied sciences. However, not every academic paper in an applied science we publish shows feasible practical solutions, even when they report record performance. As a journal with 'technology' in the title, we are comfortable working in this space. And under Fabio's leadership, we've tried to raise the awareness of the needs of the industry that lies at the endpoint of applied academic works. For example, battery papers tested in a laboratory setting should abide to certain characterization standards that would make them more likely to become practical ${ }^{1}$. Along the same line, we produced a Focus issue about nanocatalysts for industrial applications ${ }^{2}$.

Fabio has always envisioned the journal as an open forum for the exchange of ideas and sharing thoughts worth sharing. As an example, we have offered our pages to foster a high-level discussion through a series of Correspondences and Perspective articles, within the nanomedicine community

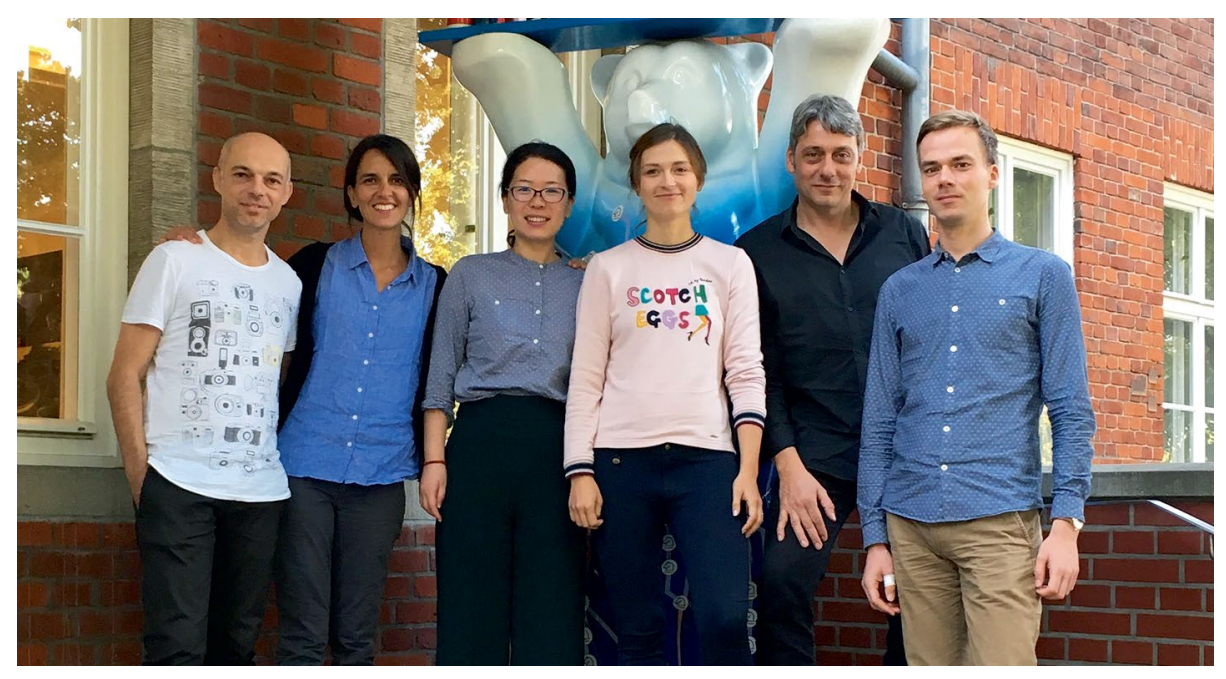

Credit: Image courtesy of Bart Verberck

throughout the years $\mathrm{s}^{3-5}$. This followed a long period of research with no major inroads to translation in clinically approved drugs from regulatory agencies and the need for reporting guidelines in the published literature.

But Fabio's most enduring legacy is the expansion of the scope of the journal to the environmental aspects of nanotechnology. He realized that one cannot talk about nanotechnology without raising awareness of the associated environmental issues. The evolution towards applications implies a more widespread production of nanomaterials. If industrial processes are not properly managed and regulated, the release of engineered nanomaterials in the environment is set to grow. As a consequence, there is the necessity to understand the potential implications of nanomaterials for the environment and human health. Research on environmental nanotechnology encompasses monitoring and remediation, eco-toxicology, as well as human acceptance of products containing nanomaterials and regulatory frameworks $s^{6}$. Micro- to nano-sized plastic particles that form from the degradation process of synthetic plastics in the environment are also a concern of environmental nanotechnology?

Fabio's interest in environmental issues has also reached communities studying sustainable agriculture ${ }^{8}$ and clean water ${ }^{9}$, producing two Insights. Nanotechnology is poised to make a big impact in clean water technologies, because of the potential to develop atomically controlled separation membranes using $2 \mathrm{D}$ materials for water desalination and water purification.

Publications about environmental nanotechnology constituted $\sim 20 \%$ of our content in 2021, compared to less than 5\% nine years ago.

No big surprise then that Fabio's passion for environmental issues has led him to Nature Water, a journal due to launch in 2023, which will cover the interaction between society and water resources beyond the role of nanotechnology.

We thank Fabio for his editorial vision and guidance in the past nine and a half years and wish him well for this new journey in his career.

Published online: 16 February 2022 https://doi.org/10.1038/s41565-022-01083-7

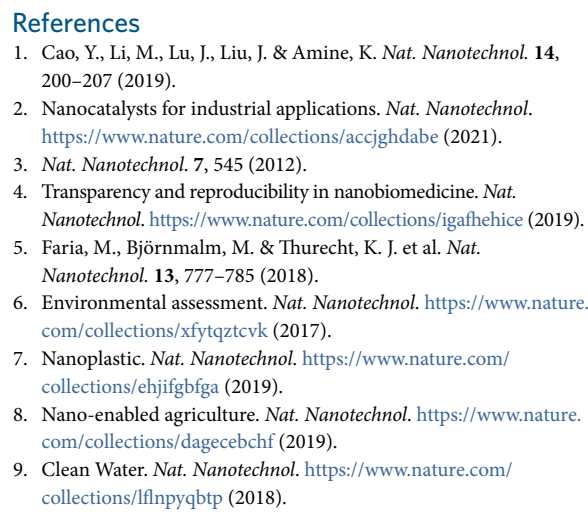

